\title{
Closing the loop: optimal stimulation of C. elegans neuronal network via adaptive control to exhibit full body movements
}

\author{
Julia Santos, Eli Shlizerman* \\ From 24th Annual Computational Neuroscience Meeting: CNS*2015 \\ Prague, Czech Republic. 18-23 July 2015
}

The Caenorhabditis elegans (C. elegans) worm is a wellstudied biological organism model. The nervous system of C. elegans is particularly appealing to study, since it is a tractable fully functional neuronal network for which electro-physical connectivity map (connectome) is fully resolved $[1,2]$. In a recent work, we succeeded in establishing a computational dynamical model for the C. elegans nervous system and showed that robust oscillatory movements in motor neurons along the body can be invoked by constant current excitation of command sensory neurons (e.g. PLM neurons associated with forward crawling) and that their activation corresponds to low-dimensional Hopf bifurcation [3]. While these first results validated the model, it is exciting to learn how the nervous system transforms its oscillatory dynamics to the muscles to support robust full body movements (e.g. forward crawling) [4]. Moreover, using methods generically applicable to other neuronal circuits, it is intriguing to understand the optimal sensory stimulations that cause these movements to persist.

We explore these questions by modeling the C. elegans musculature as a viscoelastic rod with discrete rigid segments [5], and map the neuronal dynamics such that they activate the muscles and deform the rod (Fig. 1A). When motor neuron activity stimulates muscles [2], this activation is translated into force applied to the rod, which moves in accordance with the physical properties of C. elegans. By stimulating the command PLM neurons, we establish for the first time that motor neuron dynamics are indeed producing coherent oscillatory full body movements that resemble forward crawling (Fig. 1B, videos

\footnotetext{
* Correspondence: shlizee@uw.edu

Department of Applied Mathematics, University of Washington, Seattle, WA
} 98195, USA

\section{$\int$ BioMed Central}

(0) 2015 Santos and Shlizerman This is an Open Access article distributed under the terms of the Creative Commons Attribution License (http://creativecommons.org/licenses/by/4.0), which permits unrestricted use, distribution, and reproduction in any medium, provided the original work is properly cited. The Creative Commons Public Domain Dedication waiver (http://creativecommons.org/ publicdomain/zero/1.0/) applies to the data made available in this article, unless otherwise stated.

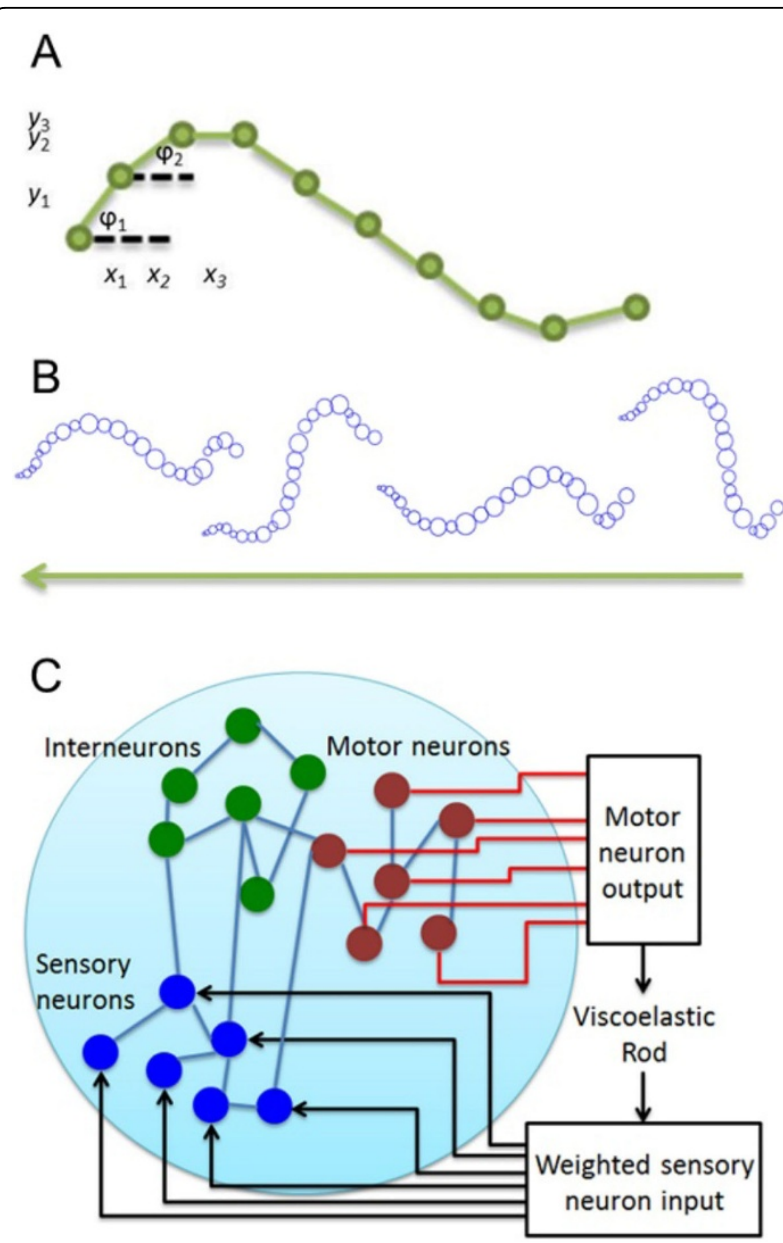

Figure $1 \mathrm{~A}$ : Structure of viscoelastic rod B: Viscoelastic rod-based simulation of C. elegans crawling during PLM excitation, videos available here C: Loop feeding transformed motor activity into sensory neurons. 
available here: http://faculty.washington.edu/shlizee/ celegans/).

We utilize our computational full body model to determine the appropriate sensory input for behavior, such as crawling, to persist after explicit external stimulation (touch) has ceased, as observed in experiments [5]. Since such persistence could be explained by a feedback loop between the environment and sensory neurons (Fig. 1C), we propose an adaptive control algorithm that extends existing recursive least squares-based algorithms (e.g. FORCE [6]). Our algorithm finds weights for synaptic input using a low-dimensional projection of motor neuron dynamics, and is capable of finding sensory input patterns that will lead to the desired movement.

Published: 18 December 2015

\section{References}

1. Varshney LR, Chen BL, Paniagua E, Hall DH, Chklovskii DB: Structural Properties of the Caenorhabditis elegans Neuronal Network. PLoS Comput Biol 2011, 7(2):1001066.

2. Sengupta P, Samuel ADT: Caenorhabditis elegans: a model system for systems neuroscience. Curr Opin Neurobiol 2009, 19(6):1-7.

3. Kunert J, Shlizerman E, Kutz JN: Low-dimensional functionality of complex network dynamics: Neurosensory integration in the Caenorhabditis elegans connectome. Phys Rev E 2014, 89(5):052805.

4. McMillen T, Williams T, Holmes P: Nonlinear Muscles, Passive Viscoelasticity and Body Taper Conspire To Create Neuromechanical Phase Lags in Anguilliform Swimmers. PLoS Comput Biol 2008, 4(8):1000157.

5. Backholm M, Ryu WS, Dalnoki-Veress K: Viscoelastic properties of the nematode Caenorhabditis elegans, a self-similar, shear-thinning worm PNAS 2013, 110(12):4528-4533.

6. Sussillo D, Abbott LF: Generating Coherent Patterns of Activity from Chaotic Neural Networks. Neuron 2009, 63(4):544-557.

doi:10.1186/1471-2202-16-S1-014

Cite this article as: Santos and Shlizerman: Closing the loop: optimal stimulation of $C$. elegans neuronal network via adaptive control to exhibit full body movements. BMC Neuroscience 2015 16(Suppl 1):O14.

\section{Submit your next manuscript to BioMed Central} and take full advantage of:

- Convenient online submission

- Thorough peer review

- No space constraints or color figure charges

- Immediate publication on acceptance

- Inclusion in PubMed, CAS, Scopus and Google Scholar

- Research which is freely available for redistribution

Submit your manuscript at www.biomedcentral.com/submit 